\title{
La experiencia de una conferencia virtual para formación profesional en Educación Matemática de primaria en Geometría
}

\author{
The experience of a virtual conference for professional training \\ in Primary School Mathematics Education in Geometry
}

\section{A experiência de uma conferência virtual para a formação profissional em Educação Matemática Primária em Geometria}

Marianela Zumbado-Castro

Universidad Estatal a Distancia San José, Costa Rica

mazumbado@uned.ac.cr

(D) https://orcid.org/0000-0002-5774-1884

Recibido - Received - Recebido: 27 / 06 / 2020 Corregido-Revised - Revisado: 07 / 08 / 2020 Aceptado - Accepted - Aprovado: 21 / 08 / 2020

DOl: https://doi.org/10.22458/ie.v22iespecial.3136

URL: https://revistas.uned.ac.cr/index.php/innovaciones/article/view/3136

\begin{abstract}
Resumen: La sistematización pretende compartir la experiencia adquirida a través del diseño y ejecución de una conferencia virtual para formación profesional con docentes de primaria en Educación Matemática, que involucró el área de Geometría, la guía de trabajo autónomo y los escenarios de conectividad, estas dos últimas como herramientas propuestas por el Ministerio de Educación Pública costarricense para enfrentar el distanciamiento social en los procesos de enseñanza y aprendizaje durante la pandemia mundial por la COVID-19, lo anterior en el marco de IV Simposio de Educación Matemática para Primaria de la Universidad Nacional (UNA). Se compartirá la respuesta a dos interrogantes ¿cómo distribuir el tiempo apropiadamente en una conferencia con ciertas características? y ¿cuáles recursos tecnológicos o digitales utilizar de acuerdo con los propósitos perseguidos tanto didácticos como de interacción con el público meta?, lo anterior, mediante la presentación de la estrategia empleada, que consistió en dividir en cuatro espacios la jornada de trabajo, con propósitos bien definidos y con recursos tecnológicos individuales para cada sección. Se concluye que el uso de dos computadoras, la atención del chat por la expositora de manera directa, la distribución por espacios de 30 minutos y la selección de los recursos tecnológicos, lograron una conferencia interactiva.
\end{abstract}

Palabras clave: formación profesional; docente de escuela primaria; aprendizaje en línea; enseñanza de las matemáticas; geometría; COVID-19

\begin{abstract}
This paper intends to share the experience acquired through the design and execution of a virtual conference for professional training with primary school teachers in Mathematics Education, which involved the area of Geometry, the autonomous work guidelines and the connectivity scenarios. These last two were tools proposed by the Costa Rican Ministry of Public Education to face social distancing in the teaching and learning processes during the global COVID-19 pandemic. The above occurred in the framework of the IV Symposium on Mathematical Education for Primary School at the National University (UNA). The answer to two questions will be shared: (i) How to allocate time appropriately in a conference with certain characteristics? (ii) What technological or digital resources to use in accordance with the purposes pursued, both didactic and of interaction with the target audience? These answers obtained are shared by presenting the strategy used, which consisted in dividing the workday into four spaces, with well-defined purposes and with individual technological resources for each section. It is concluded that the use of two computers, the lecturer directly attending to the chat, the distribution of 30-minute spaces and the selection of technological resources, achieved an interactive conference.
\end{abstract}

Key Words: professional training; primary school teacher; online learning; math teaching; geometry, COVID-19 
Resumo: Sistematização visa compartilhar a experiência adquirida através da concepção e implementação de uma conferência virtual de formação profissional com professores primários em Educação Matemática, que envolveu a área de Geometria, o guia de trabalho autônomo e cenários de conectividade, estes dois últimos como ferramentas propostas pelo Ministério da Educação Pública da Costa Rica para abordar o distanciamento social nos processos de ensino e aprendizagem durante a pandemia global pela COVID-19, isto no âmbito do IV Simpósio sobre Educação Matemática para o Ensino Primário da Universidade Nacional (UNA). A resposta a duas perguntas será compartilhada: como distribuir o tempo adequadamente em uma conferência com determinadas características? e que recursos tecnológicos ou digitais utilizar de acordo com os objetivos perseguidos, tanto didáticos como em termos de interação com o público alvo? Isto será feito através da apresentação da estratégia utilizada, que consistiu em dividir o dia de trabalho em quatro espaços, com objetivos bem definidos e recursos tecnológicos individuais para cada seção. Concluiu-se que o uso de dois computadores, a atenção do bate-papo pelo expositor de forma direta, a distribuição por espaços de 30 minutos e a seleção dos recursos tecnológicos, conseguiram uma conferência interativa.

Palavras-chave: formação profissional; professor primário; aprendizagem on-line; ensino de matemática; geometria, COVID-19

\section{INTRODUCCIÓN}

El propósito del documento es compartir con otros docentes y académicos la experiencia generada al diseñar e impartir la conferencia virtual "Geometría: Guía de trabajo autónomo y escenarios de conectividad", que formó parte del IV Simposio de Educación Matemática en Primaria (IV SEMP), de la Escuela de Matemáticas de la Universidad Nacional de Costa Rica, que debido a la situación sanitaria mundial generada por el virus responsable de la COVID-19 en el 2020 se realizó en una modalidad virtual.

Compartir los aprendizajes de esta experiencia virtual, cobró importancia debido a que los docentes y los académicos que se desempeñan en la presencialidad no estabamos preparados para enfrentar los retos de realizar procesos de formación docente a distancia (Varguillas y Bravo, 2020), porque en estos espacios en línea los vínculos de comunicación son diferentes a los propios de la presencialidad, la tradición socioconstructivista está ausente o limitada por el distanciamiento físico y transformada por los recursos tecnológicos, donde la ausencia de articulación entre un propósito educativo y la tecnología, se convierte en un obstáculo para los procesos de enseñanza y aprendizaje (Janssen, Knoef y Lazonder, 2019; Sosa, Salinas y de Benito, 2018; Zumbado-Castro, 2019).

Entonces, al dirigir una actividad virtual de aprendizaje sincrónica surgieron algunas preguntas, entre ellas ¿cómo distribuir el tiempo apropiadamente en una conferencia con ciertas características? y ¿cuáles recursos tecnológicos o digitales utilizar de acuerdo con los propósitos perseguidos tanto didácticos como de interacción con el público meta?

Por tanto, el propósito del ensayo es compartir aciertos y desaciertos de la estrategia seleccionada para efectuar una conferencia con docentes de primaria, con el objetivo de contribuir al fortalecimiento de las habilidades de la persona docente en el manejo de actividades de mediación pedagógica en un entorno virtual y exponer ante la comunidad científica la estrategia empleada para su juzgamiento y optimización.

\section{DESARROLLO DEL TEMA}

\section{Conferencia y contexto}

De acuerdo con el Diccionario de la Lengua Española (2001), una conferencia es una exposición oral sobre un tema a un grupo numeroso de personas de manera simultánea, por lo general, este tipo de actividad es presencial. 
Sin embargo, en este contexto de pandemia, se hace necesaria una transformación de la modalidad presencial a la virtual, para dar continuidad a los procesos de formación y comunicación, lo que implica adecuaciones metodológicas y tecnológicas. Ahora una conferencia se debe realizar de forma remota, lo que cambia el escenario, se emplea un dispositivo con conexión a internet, se usa el micrófono, los parlantes o los audífonos, donde la interacción se hace mediante un chat o con cortas intervenciones verbales e individuales (Zumbado-Castro, 2020).

Por esa razón, es necesario que la persona que expondrá en una conferencia virtual prepare un guion, que incluya cada cierta cantidad de minutos actividades de interacción con objetivos pedagógicos claros que contribuyan en el abordaje del tema o los contenidos tratados y que no se conviertan en obstáculos o distractores del propósito que persigue la sesión (Janssen et al., 2019).

Es importante señalar que las actividades de interacción pueden incluir: aplicaciones gratuitas, espacios de diálogo y discusión dirigida, encuestas en línea, videos y la exploración de sitios en la web, entre muchas otras.

Respecto al contexto específicamente, la invitación al simposio se extiendió a nivel nacional, mediante redes sociales, sin embargo tuvo un alcance internacional, debido a que habían participantes de otras latitudes. El cupo era inicialmente para 200 personas, en pocas horas se había cerrado la inscripción debido a que alcanzó un registro de 230 formularios de personas interesadas.

Estaba dirigida a personal docente de escuela primaria, sin embargo, se verificó la participación de docentes de secundaria y supervisores regionales de la enseñanza de las Matemáticas, denominados asesores pedagógicos. La conferencia "Geometría: Guía de trabajo autónomo y escenarios de conectividad" contó con la participación de 136 personas, es importante indicar que el promedio del evento fue de 140.

Cada conferencia debía abarcar entre una hora con treinta minutos hasta las dos horas máximo. Se contó con la participación de docentes de diferentes regiones educativas de Costa Rica y países como: Brasil, Chile, Colombia, Perú y España. Recibieron certificación 125 docentes, quienes cumplieron con una participación de $80 \%$ de las 12 horas totales del evento, los datos fueron suministrados por la Organización del IV SEMP, 2020.

La conferencia abordó tres temáticas básicas: un conocimiento matemático geométrico, específicamente la simetría, la guía de trabajo autónomo (GTA) y los escenarios de conectividad, todas estas nociones pertenecientes al MEP y las dos últimas elaboradas por esa entidad para el contexto del COVID-19.

Antes de continuar, es necesario conocer de acuerdo con la definición asignada por el MEP, qué es una GTA y cuáles son los escenarios de conectividad.

De acuerdo con MEP (2020a), la GTA corresponde a una plantilla que sustituye la mediación pedagógica presencial, se incluyen tres momentos: planificación, autorregulación y evaluación, que se ampara en la Dimensión 1, denominada: "Maneras de pensar" específicamente en la habilidad "Aprender a aprender" de la política curricular vigente denominada "Educar para una nueva ciudadanía", aprobada en 2015.

Además, el ente ministerial indica que la mediación pedagógica (estrategia metodológica) debe ser la que se plantea en los programas oficiales de la asignatura. Su construcción debe considerar un Diseño Universal del Aprendizaje (DUA), por tanto, se espera que toda persona estudiante, en cualquier contexto, logre realizar las tareas incluidas en el documento, de manera independiente, a partir del uso de conocimientos previos y de instrucciones claras y precisas.

El propósito de una GTA es la ejecución de actividades de aprendizaje vinculadas con habilidades específicas (objetivos o competencias) de una asignatura y finalmente, recolectar una evaluación del proceso de aprendizaje (autoevaluación). La guía puede ser desarrollada en un lapso de entre 40 y 60 minutos (MEP, 2020a). 
Respecto a los escenarios de conectividad el MEP (2020b) definió cuatro, los cuales corresponden a: el primero, dispositivo y acceso a internet en casa; el segundo, dispositivo y acceso limitado o reducido; el tercero, dispositivo sin acceso a internet y finalmente, sin dispositivo.

Es importante señalar que el propósito de la conferencia fue mostrar cómo incluir en una GTA la estrategia metodológica de resolución de problemas, para el abordaje de las siguientes habilidades específicas de Geometría para cuarto grado de manera integrada:

22. Identificar los ejes de simetría de una figura.

23. Ubicar un punto homólogo a otro respecto a una recta.

24. Trazar una figura simétrica a otra respecto a una recta.

25. Estimar la distancia de un punto al eje de simetría (MEP, 2012, p. 204)

Así como proponer algunas ideas para ajustar una GTA básica a los escenarios de conectividad que plantea el MEP (2020b), para conseguir este propósito se planteó la siguiente estrategia.

\section{Estrategia de mediación para una conferencia virtual}

El tiempo máximo disponible fue de dos horas, se planificó la conferencia en cuatro espacios de 30 minutos máximo cada uno y sus respectivos recursos tecnológicos. A continuación, se describen las instrucciones generales, el propósito, el desarrollo, así como las aplicaciones empleadas en cada sección.

\section{Instrucciones generales}

A continuación se detallan las instrucciones que se dictaron, las cuales fueron fundamentales para la ejecución y nivel de interactividad conseguido, por tanto, se considera que esta sección puede contribuir en la conformación de un protocolo de ejecución de conferencias virtuales.

Se dictaron dos instrucciones: la primera relacionada con el chat y la segunda con el uso de un dispositivo adicional para participar de actividades en línea vinculadas con sitios web, aplicaciones y lectura de códigos de respuesta rápida conocidos como código QR, por sus siglas en inglés. Es importante indicar que el QR es un código de barras bidimensional que se usó en la conferencia para almacenar enlaces a sitios web y agilizar el acceso a ellos (Gómez-Gonzalvo, Atienza y Mir, 2015).

Se indicó a los participantes que la expositora requería que a través del chat comprobaran si se estaba dando una correcta comunicación durante toda la conferencia, por tanto, se debía responder por parte de alguno de los participantes a cada pregunta que se planteaba, entre ellas: ¿se escucha?, ¿existe alguna duda?, ¿se compartió la pantalla? o ¿se había considerado esa posibilidad?, ¿hay algún desacuerdo o hay alguna propuesta?, entre otras. Es importante indicar que la expositora utilizó dos portátiles para poder tener el chat abierto con texto ampliado para atender las consultas con mayor facilidad.

Respecto al dispositivo adicional, se indicó que al utilizar el computador o una tableta también se debía disponer de un celular para facilitar el acceso a algunas aplicaciones que se emplearían en el desarrollo del tema. Además, se explicó que para facilitar el acceso a algunos sitios se insertaron códigos QR para evitar la digitación de las direcciones electrónicas. Posteriormente, se inició con las actividades. 


\section{Espacio 1. Geometría: la simetría}

El propósito de este espacio fue modelar la primera etapa de acuerdo con la estrategia metodológica de resolución de problemas establecida por el MEP (2012) para organizar las lecciones de Matemáticas, denominada "El aprendizaje del conocimiento" (p. 41), que consiste a su vez, en cuatro momentos: el primero, "la propuesta de un problema" (p. 41), se realizó mediante una imagen (Figura 1), en la cual se debía determinar ¿cuántos cuadrados de cada color se requerían para completar la otra mitad de la imagen?

Figura 1. Problema inicial



Fuente: Cokitos juegos educativos online (2020). https://www.cokitos.com/

Durante el segundo momento, denominado "trabajo estudiantil independiente" (MEP, 2012, p. 41), se pretendió que la persona estudiante o participante enfrentara el problema, se recurrió a una aplicación gratuita en línea llamada QUIZIZZ, la cual permitió responder cuatro preguntas, cada una con 30 segundos de tiempo. Se registraron las respuestas y se visualizó el avance de los participantes a través de la página web de la aplicación. 
Figura 2. Visualización de la aplicación por el participante en el dispositivo adicional y registro para el conferencista en el sitio web de la aplicación.
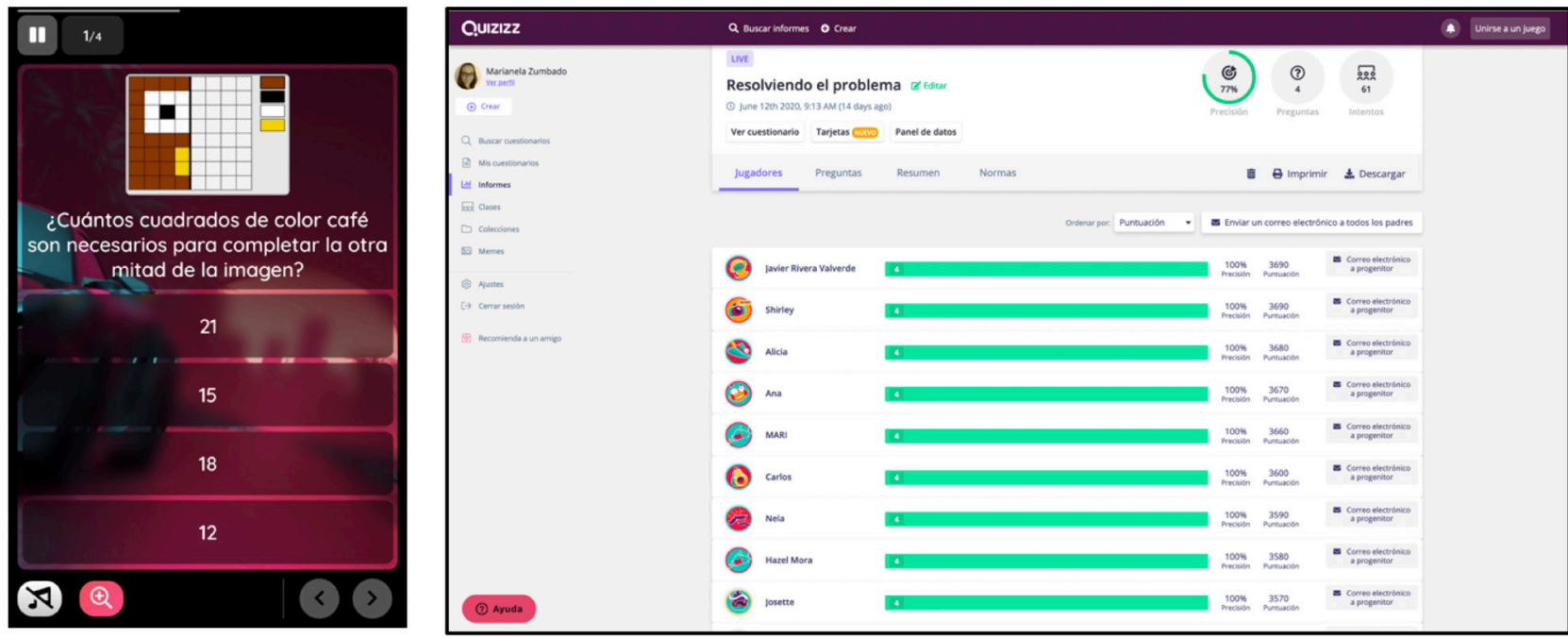

Fuente: Actividad realizada en el IV SEMP 2020. Elaboración propia (2020).

Es importante indicar que de las 136 personas participantes en la conferencia, en esta primera actividad se registró la intervención de 61 participantes, con $77 \%$ de efectividad en el acierto de las preguntas planteadas. Los datos fueron recolectados y tabulados por la misma aplicación QUIZIZZ de manera automática, que mostró el proceso de cálculo en tiempo real como se muestra en la Figura 2. Posteriormente, se abrió un espacio para que tres docentes explicaran ¿cómo hallaron la respuesta a cada pregunta?, se enfatizó en la estrategia de resolución y no en la respuesta.

Las acciones anteriores pretendieron modelar el tercer momento de la estrategia metodológica de resolución de problemas, denominado "discusión interactiva y comunicativa" (MEP, 2012, p. 41).

Para concluir con el proceso de modelado de los cuatro momentos de una lección de Matemáticas, de acuerdo con la propuesta del MEP (2012), en la conferencia se procedió a realizar el "cierre o clausura" (p. 41) para formalizar los conocimientos, esto consistió en el proceso de exponer las definiciones de los conceptos involucrados de manera formal, pero vinculado con el problema trabajado, entre ellos: figura simétrica, eje de simetría, puntos homológos y distancia de un punto al eje de simetría.

\section{Espacio 2. GTA}

La intención de este espacio fue exponer brevemente las ideas principales que el MEP (2020a) plantea sobre la GTA, con el propósito de nivelar los conocimientos sobre la plantilla que poseían los participantes nacionales e internacionales.

Se abordó la política curricular, específicamente la dimensión involucrada y la habilidad, la función de la GTA, las implicaciones de la transformación curricular que indica que la persona estudiante es el centro del quehacer educativo (MEP, 2015). Además, se abordó la metacognición, la importancia de la secuencialidad, concatenación y gradualidad, así como la correspondencia con los programas de la asignatura, en este caso Matemáticas y la metodología que plantean (MEP, 2020a). Por esto último, era necesario modelarla previamente. Además, se puntualizó en la importancia de los conocimientos previos, la motivación y el contexto, ideas presentadas mediante la Figura 3. 
Figura 3. Lamina de la presentación sobre la GTA.

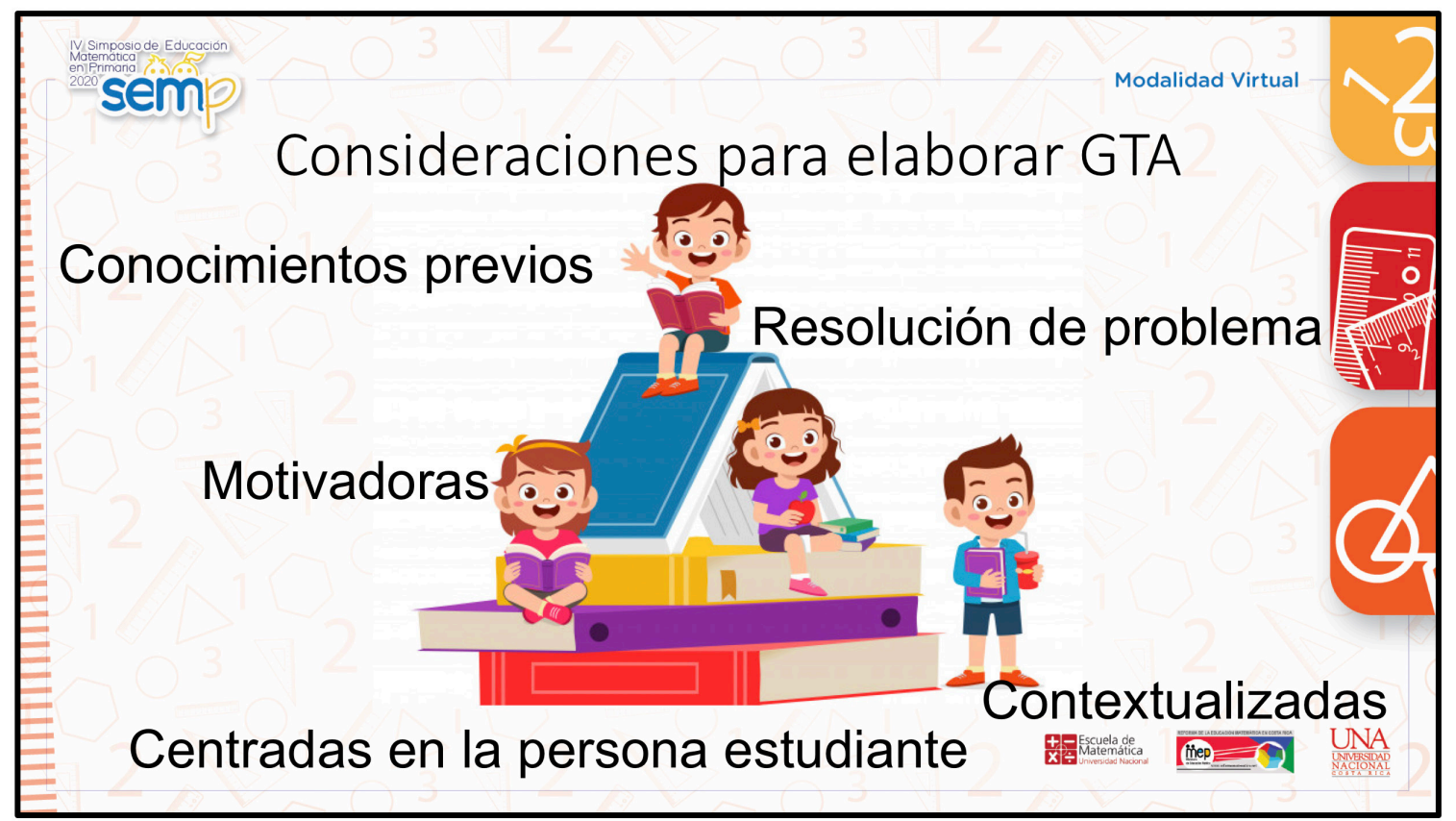

Fuente: Presentación utilizada en el IV SEMP 2020. Elaboración propia (2020).

Concluida la exposición teórica, se procedió a presentar en formato PDF una GTA para cuarto grado, que incluía el problema que se había resuelto previamente mediante QUIZIZZ y que propiciaba las habilidades específicas citadas y los conocimientos que se formalizaron.

Además, dentro de la sección "Actividades para introducir el nuevo conocimiento" que pertenece a la GTA, se incluyeron los cuatro momentos que se plantean en los programas de Matemáticas para la organización de la lección, que fueron enunciados previamente y que se recapitulan aquí: propuesta de un problema, trabajo estudiantil independiente, discusión interactiva y comunicativa y, finalmente, la clausura o cierre (MEP, 2012).

A partir de este momento, se sometió a la opinión de los participantes la propuesta presentada en PDF, mediante una encuesta en línea de Google Form, que además se facilitó mediante un código QR y que contenía las siguentes cinco preguntas:

1. ¿Modificaría la GTA que presentó la expositora?

2. ¿Cuál aspecto modificaría de la GTA?

3. Si marcó "otro" indique cuál aspecto modificaría en la GTA.

4. ¿Cómo efectuaría la modificación en la GTA?

5. ¿La GTA reconstruida propiciará los procesos matemáticos?

En un periodo menor a diez minutos, se recibieron las respuestas de 40 participantes y se mostraron directamente de la página web los resultados. 
Figura 4. Registro de respuestas de los participantes en Google Form

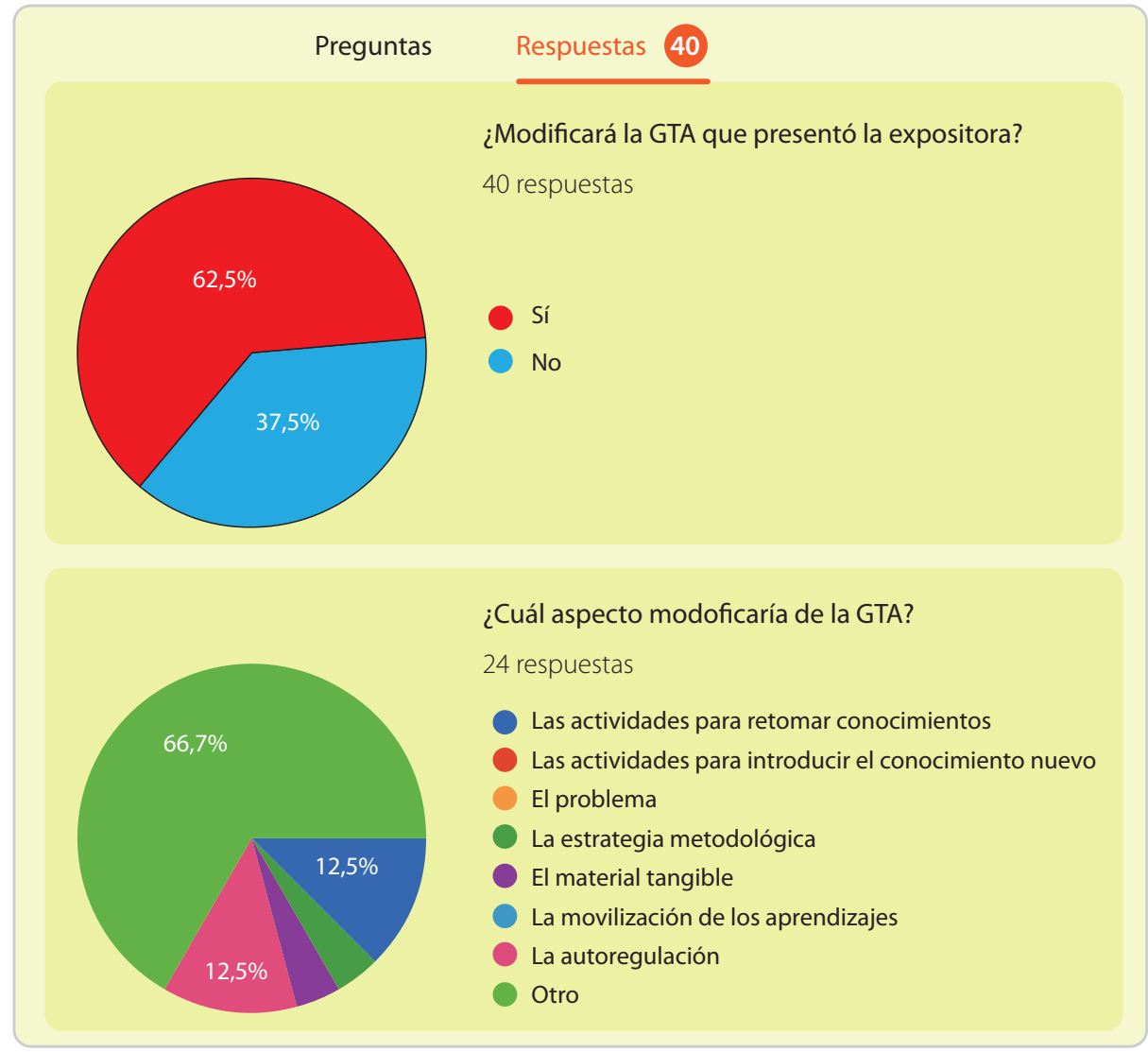

Fuente: Elaboración propia (2020).

Además, durante la recolección de los datos se mostró cómo se modificaban los porcentajes de las respuestas del cuestionario en vivo y se discutieron algunas de las propuestas de modificación indicadas por los participantes en la respuesta a la pregunta 2, 3 y 4. (ver figura 4).

\section{Espacio 3. Escenarios de conectividad}

Se prosiguió con un rápido recorrido por la definición de los escenarios de conectividad que propone el MEP (2020b), sin embargo, en la conferencia se afirmó que en realidad solamente existen dos escenarios, debido a que trabajar en un dispositivo móvil guías de varias páginas y de todas las asignaturas se convierte para la persona estudiante en una limitante por al menos dos razones: el cansancio que implica leer de un dispositivo móvil mucho tiempo (fatiga visual, sequedad ocular, problemas de sueño y aumento de miopía) y las dificultades para realizar ciertas actividades desde una pantalla pequeña, que además tiene poca o limitada conectividad (Sans, Cardona y Pérez, 2016). 
Figura 5. Lamina de la presentación sobre los escenarios de conectividad.

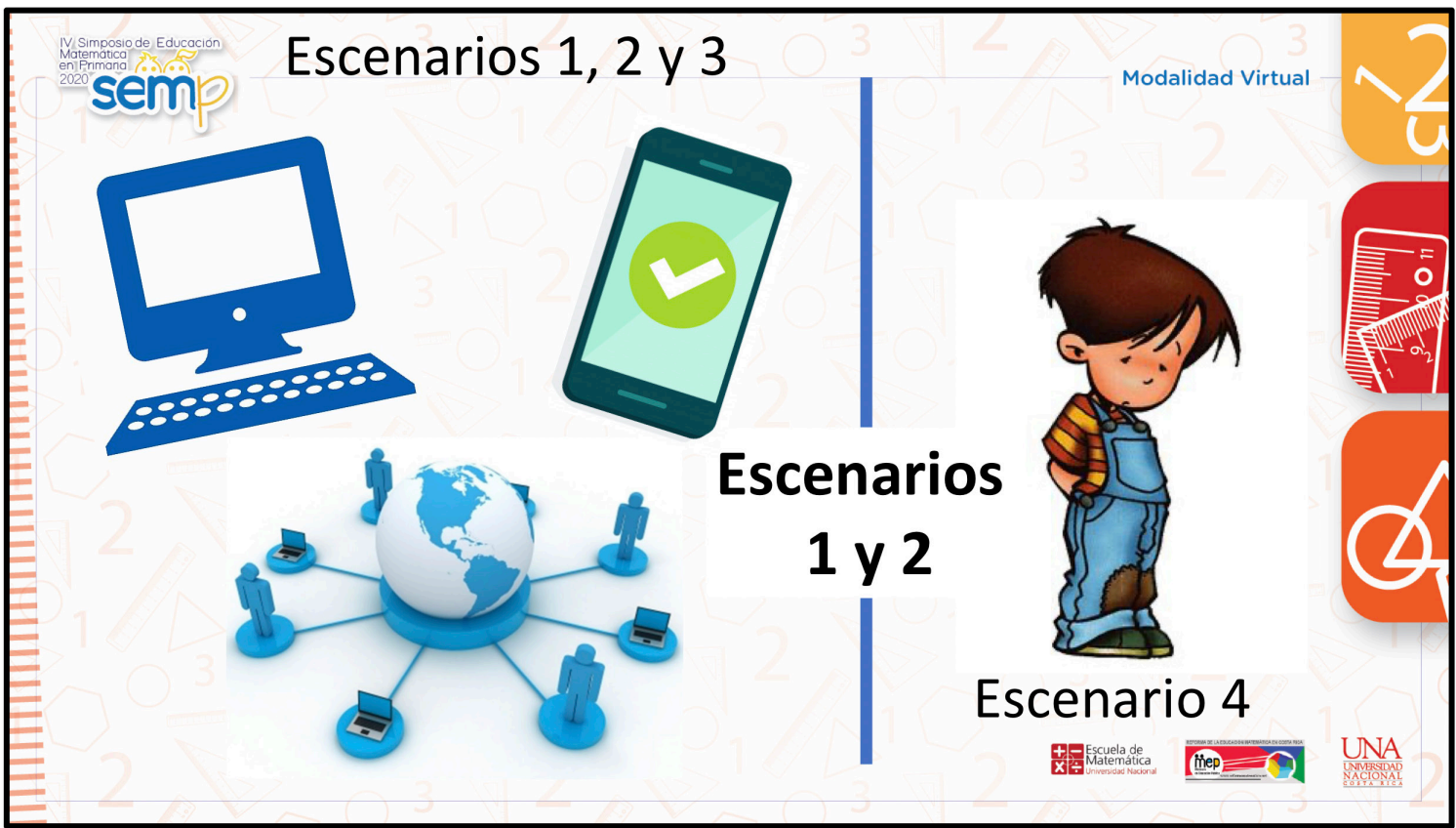

Fuente: Presentación utilizada en el IV SEMP 2020. Elaboración propia (2020).

Posteriormente, se mostró en formato PDF una GTA para cuarto grado modificada, con pequeños ajustes que incluyeron variaciones relacionadas con los dos escenarios de conectividad enfatizados en la exposición (Figura 5). Si se considera lo anterior y el acceso universal que persigue el MEP (2018), es necesario que la persona docente planifique cada tarea considerando un acceso equitativo al conocimiento, por tanto, cada guía debe poseer los mismos elementos básicos de aprendizaje para cualquier población, en cualquier escenario de conectividad y en cualquier condición económica (MEP, 2018), por tanto, se diseñó la GTA para un escenario sin dispositivo, pero se amplió con algunos componentes digitales para el escenario 1,2 y 3 .

Se cerró este espacio con un chat para un intercambio de ajustes, sugerencias o modificaciones.

\section{Espacio 4. Movilización y autorregulación}

Esta fue la última sección de la conferencia, en la que se inició con la exploración de un cuestionario en línea, esta vez en la plataforma TEAMS ${ }^{\circledR}$ de Microsoft. Se indicó que el propósito era hacer una movilización de los aprendizajes, de manera análoga a la segunda etapa del aprendizaje que propone el MEP (2012) para la organización de las lección de Matemáticas.

La actividad consistió en llenar un cuestionario en línea que tenía el formato de una prueba corta, calificada de manera automática y vinculada de manera directa con la exposición previa y a la cual se accedió mediante un código QR. Durante la ejecución se mostró la modificación de la base de datos en tiempo real y se comentaron algunas respuestas (Figura 6). Sin embargo, en esta oportunidad con menos datos registrados, esto debido a que en el chat habían constantes mensajes que indicaron problemas para acceder al cuestionario y manifestaciones de angustia por no poder ingresar. Se registraron únicamente 19 formularios con una puntuación media de acierto de 6.2 de 10 . 
Figura 6. Visualización del cuestionario en el dispositivo móvil y registro en la plataforma TEAMS ${ }^{\oplus}$.
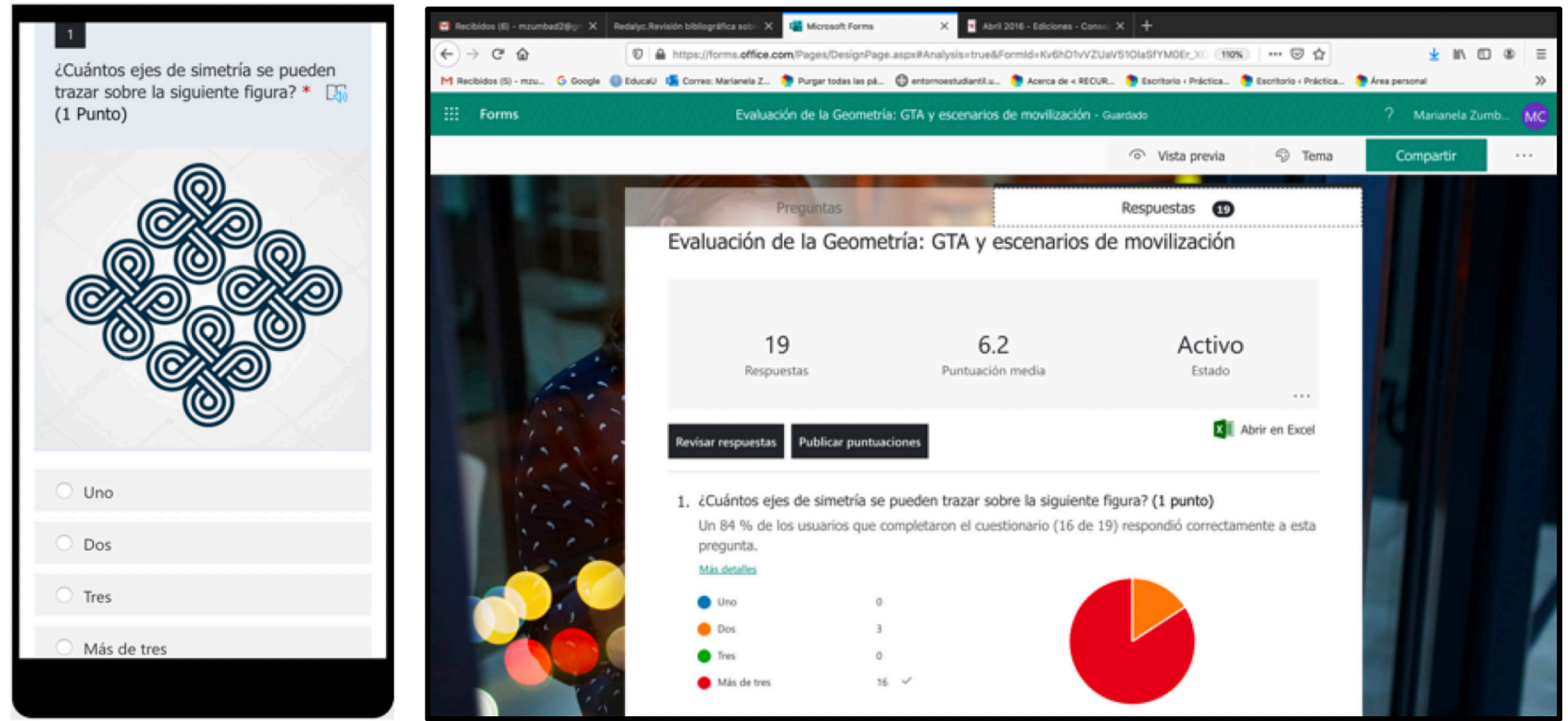

Fuente: Datos de la página de Form (2020). Elaboración propia (2020).

Se cerró este espacio con algunas recomendaciones generales, entre ellas incluir preguntas básicas sobre conocimientos en las autoevaluaciones, acciones que involucren a la familia y los conocimientos adquiridos, ítems con diferentes niveles de complejidad y de pensamiento inverso. Así como utilizar diferentes herramientas para la autoevaluación (encuesta en línea) y personalizar el diálogo y las aplicaciones en la medida de las posibilidades, con mensajes personales como: "Gracias. Saludos de su maestra Marianela Zumbado". Posteriormente, la conferencia finalizó cuando la anfitriona del comité organizador sistematizó las últimas consultas, los agradecimientos y dio por concluida la sesión.

Después de haber descrito los espacios que conformaron la conferencia, se procederá a indicar algunos aspectos positivos y negativos de la experiencia.

\section{Aciertos y desaciertos}

Dentro de la experiencia se pueden considerar al menos cinco aciertos. El primero, utilizar dos computadoras para mantener un contacto cercano con los participantes a través del chat, al respecto de la comunicación con el público, Varguillas y Bravo (2020) indican que esto es fundamental "dar respuestas oportunas y claras ante las dudas" (p. 230), debido a que se promueve la interacción entre la persona expositora y los participantes.

El segundo acierto fue la distribución de la conferencia en espacios de 30 minutos aproximadamente porque se favoreció la interacción, las actividades simularon un taller y eso promovió el intercambio entre la persona expositora y el público. El tercer acierto fue establecer un propósito para cada espacio, así como un recurso tecnológico que cumpliera una funcion didáctica específica: resolución de problemas, valoración y discusión de una GTA y evaluación del aprendizaje adquirido. Estos dos aciertos, se relacionan con los resultados de Janssen et al. (2019), que indican que la integración de la tecnología con la pedagogía y el contenido genera resultados positivos cuando son planificados de manera detallada. 
El cuarto acierto fue haber realizado pruebas con todas las aplicaciones de manera previa, sin embargo, esto no evitó inconvenientes que se puntualizarán más adelante. Al respecto, Sosa et al. (2018) sugieren que es recomendable interactuar con los recursos tecnológicos con el objetivo de adquirir destrezas en su manejo y con ello anticipar inconvenientes, además señalan que es importante "entender que la tecnología no es el fin, sino un medio para ayudar a mejorar los procesos de enseñanza y aprendizaje" (p. 79).

También, el estudio y participación en eventos similares, se considera el quinto acierto, debido a que se adquirió alguna experiencia desde la observación no participante.

Respecto a los desaciertos, el primero fue no lograr compartir el audio de un video, posteriormente, se descubrió que fue un error al iniciar la sesión en $\mathrm{ZOOM}^{\circledR}$, por tanto, la recomendación de Sosa et al. (2018) es pertinente otra vez. El segundo desacierto fue comunicar con anticipación que en el último espacio "Movilización y autoregulación" se iniciaría con una prueba corta, porque esto pudo incidir en el reducido número de las respuestas al cuestionario en TEAMS ${ }^{\circledR}$, sobre el tema Flores y López (2019) afirman que en las evaluaciones en línea se deben considerar dos aspectos: la retroalimentación oportuna y la forma de calificar las actividades, por tanto, el no comunicar que cada ítem tenía como valor un punto y que el formulario iba a generar un comentario que complementaba la opción seleccionada, pudo impactar también en el bajo número de respuestas obtenidas.

El tercer desacierto se asocia con esta última plataforma, debido a que existió un problema técnico de acceso, que aún no se logra determinar, se sospecha que al ser una plataforma corporativa el ingreso de "invitados" por el enlace generó algunas dificultades.

Finalmente, el uso de códigos QR se puede ubicar en un punto medio entre acierto y desacierto, porque para algunos participantes fue muy fácil ingresar a través del escaneo, sin embargo, para otros, fue necesario compartir el enlace, por tanto, no es claro cuál fue el impacto real de su uso y si esto tuvo relación con la falta de habilidades informáticas de algunos de los participantes (Vargillas y Bravo, 2020).

\section{SÍNTESIS Y REFLEXIONES FINALES}

Al reflexionar sobre la temática "Educación en tiempos de pandemia: estrategias para la mejora de lo procesos educativos", se puede indicar que en el contexto del COVID-19 ha sido necesario reestructurar la táctica empleada en una conferencia presencial para atender mediante una nueva estrategia los retos de una conferencia en la virtualidad, con el objetivo de garantizar procesos educativos de igual o mejor calidad, donde aún sea posible la comunicación e interacción con los participantes.

A modo de síntesis se presenta la siguiente tabla que sistematiza esa nueva estrategia empleada en la conferencia por cada espacio, lo que permitirá una respuesta directa a las preguntas planteadas en la introducción ¿cómo distribuir el tiempo apropiadamente en una conferencia con ciertas características? y ¿cuáles recursos digitales utilizar de acuerdo con los propósitos tanto didácticos como de interacción con el público meta? 
TABLA 1

Síntesis del porcentaje de interacción por espacios, propósitos y recurso tecnológico utilizado.

$\begin{array}{cccc}\text { Espacio } & \text { Propósito } & \text { Recurso tecnológico } & \text { Interacción } \\ 1 & \text { Resolver un problema } & \text { QUIZIZZ } & 45 \% \\ 2 & \text { Recolectar la opinión sobre la primera versión de la GTA } & \text { Encuesta de Google Form } & 30 \% \\ 3 & \begin{array}{c}\text { Discutir sobre las posibles mejoras de la segunda ver- } \\ \text { sión de la GTA }\end{array} & \text { Chat de ZOOM } & \\ 4 & \text { Ejemplarizar una medición del aprendizajes on-line } & \text { Cuestionario de Microsoft TEAMS } & \\ 4 & 15 \%\end{array}$

Fuente: Elaboración propia.

Al repensar sobre la experiencia, se concluye que el uso de dos computadoras, la atención del chat por la expositora de manera directa, la distribución por espacios de 30 minutos y la selección de los recursos tecnológicos, lograron una conferencia interactiva, esta afirmación está respaldada en los datos de la tabla 1.

Además, se logró modelar las dos etapas y los cuatro momentos que plantea la estrategia metodológica de resolución de problemas según el MEP (2012) y se propiciaron al menos cuatro espacios durante la conferencia con algún porcentaje de interactividad, aunque no es posible establecer qué obstaculizó una mayor participación, se puede especular que las razones están vinculadas con la conectividad, la no disponibilidad de más de un dispositivo o limitadas habilidades tecnológicas, un ejemplo de esto último es que se requería de cierto conocimiento sobre el uso e instalación de aplicaciones, como un lector QR o el acceso en línea a sitios que requerían el ingreso de códigos numéricos como QUIZZIZ (Sosa et al. 2018; Vargillas y Bravo, 2020).

En las condiciones dadas, se generó algún nivel de discusión entre el público meta y la expositora, a través de la intervención mediante audio y video de tres docentes, en cada oportunidad se habilitó el micrófono para tal efecto (espacio 1 y 3 ).

La experiencia favoreció la reconstrucción de la GTA propuesta inicialmente, a través del trabajo colaborativo, una evidencia de esto se encuentra en el video, donde tres docentes hacen aportes para optimizar el documento a través de las siguientes sugerencias: la inclusión de "caritas" en la rúbrica para establecer los niveles de logro, la adaptación para personas con necesidades educativas especial (NEE) que presentan alguna discapacidad visual y el uso del tangrama como material de apoyo.

Un producto de esta experiencia son nuevas preguntas de investigación que las Ciencias de la Educación deben tratar de responder, entre ellas: ¿cómo establecer el nivel de logro sobre los aprendizajes adquiridos por la persona participante de una conferencia virtual?, ¿cuál es el impacto de los conocimientos adquiridos mediante una conferencia virtual en la formación profesional o en el campo de acción del participante?, ¿cuál es el impacto de una conferencia virtual en comparación con una presencial? Y finalmente ¿cuáles características debe poseer una estrategia para ejecutar una conferencia virtual exitosa cuyo propósito sea contribuir en la formación profesional?

Para finalizar, el propósito del ensayo fue compartir la experiencia vivida, sistematizando la estrategia empleada para diseñar y ejecutar una conferencia virtual para docentes de escuela primaria que contribuyera en su formación profesional. Así, se somete a la comunidad científica para su juzgamiento y optimización, en momentos donde cada actuación de los docentes es experimental, debido a que muchos de nosotros no estabamos preparados para asumir los procesos de enseñanza y aprendizaje de manera remota. Se invita a observar la ejecución de la estrategia descrita en la grabación de la conferencia, solo se debe escanear el código QR. 
Janssen, N., Knoef, M. y Lazonder, A. (2019) Technological and pedagogical support for preservice teachers' lesson planning, Technology, Pedagogy and Education, 28(1), 115-128. DOI: 10.1080/1475939X.2019.1569554.

Flores, K. y López, M. (enero, 2019). Evaluación de cursos en línea desde la perspectiva del estudiante, un análisis de métodos mixtos. Perspectiva Educacional, 58(1). Recuperado de https://dialnet.unirioja.es/servlet/autor?codigo $=1769963$

Gómez-Gonzalvo, F., Atienza, R. y Mir, M. (setiembre, 2015). Revisión bibliográfica sobre usos pedagógicos de los códigos QR. @tic.revista d'innovació educativa, (15). Recuperado de https://www. redalyc.org/pdf/3495/349543461004.pdf

Ministerio de Educación Pública. (2020a). Guía de Trabajo Autónomo Plantilla Tercer Ciclo y Educación Diversificada. San José: Ministerio de Educación Pública. Recuperado de https:// aulavirtualabierta.mep.go.cr/seccion/gta-plantilla-tercer-ciclo-y-educacion-diversificada/ gta-plantilla-tercer-ciclo-y-educacion-diversificada/

Ministerio de Educación Pública. (2020b). Orientaciones para el apoyo del proceso educativo a distancia. San José: Ministerio de Educación Pública. Recuperado de https://www.mep.go.cr/sites/default/ files/orientaciones-proceso-educativo-distancia_0.pdf

Ministerio de Educación Pública. (2018). Diseño Universal para el Aprendizaje (DUA). San José: Ministerio de Educación Pública. Recuperado de http://www.drea.co.cr/sites/default/files/Contenido/09

Ministerio de Educación Pública (2015). Fundamentación pedagógica de la transformación curricular. San José: Ministerio de Educación Pública. Recuperado de https://www.mep.go.cr/ educar-para-nueva-ciudadania

Ministerio de Educación Pública. (2012). Programas de Estudio de Matemáticas. I, II y III Ciclos de la Educación General Básica y Ciclo Diversificado. San José: Ministerio de Educación Pública.

Real Academia Española. (2001). Diccionario de la lengua española (22. ${ }^{a}$ ed.). Madrid, España: Real Academia Española. Recuperado de https://www.rae.es/

Sans, M., Cardona G. y Pérez, E. (abril, 2016). Cómo afectan las pantallas electrónicas al sistema visual. Gaceta de optometría y óptica oftálmica, (513). Recuperada de https://dialnet.unirioja.es/servlet/ articulo?codigo $=5461139$

Sosa, E. Salinas, J. y de Benito, B. (marzo, 2018). La observación reflexiva y su papel en la incorporación de Tecnologías Emergentes en el aula. Areté, 4(7). Recuperado de https://dialnet.unirioja.es/ servlet/articulo?codigo $=6833696$

Varguillas, C. y Bravo, P. (enero, 2020). Virtualidad como herramienta de apoyo a la presencialidad, análisis desde la mirada estudiantil. Revista de Ciencias Sociales, 26(1). Recuperado de https://dialnet. unirioja.es/servlet/articulo?codigo $=7384416$

Zumbado-Castro, M. (2019). Evidencias sobre algunas tendencias pedagógicas y didácticas presentes en los programas de estudio de matemáticas costarricense. Uniciencia, 33(2), 27-41. https://doi. org/10.15359/ru.33-2.3

M. Zumbado-Castro. ( $1^{\circ}$ de junio de 2020). Inédito: Las 3 enseñanzas de una lección de matemáticas en el escenario virtual. [Mensaje en un blog]. Recuperado de https://blog.ciaem-redumate.org/ inedito-las-3-ensenanzas-de-una-leccion-de-matematicas-en-el-escenario-virtual/ 